\title{
MAC/PHY Cross-Layer Design and Analysis for Multiple Packet Detector MIMO
}

\author{
Sanaz Barghi Hamid Jafarkhani Homayoun Yousefi'zadeh \\ Department of EECS \\ University of California, Irvine \\ [sbarghi, hamidj, hyousefi] duci.edu
}

\begin{abstract}
Coping with collisions is one of the biggest challenges in the design of MAC algorithms for wireless networks. Recent advances in MIMO communications have provided the possibility of decoding colliding packets. In this paper, we introduce a new MIMO cross-layer PHY/MAC design that is capable of combating collisions through the use of a multiple packet detection technique. Analytical and simulation results show that the proposed MAC design can considerably improve the throughput of a WLAN operating over lossy links.
\end{abstract}

\section{INTRODUCTION}

In recent years, demands for high-quality high-speed wireless communications have been increasing. Among wireless local area networks (WLANs), IEEE802.11 [1] family of standard have become more popular than most if not all of the other competing standards. Up until now, the main design philosophy behind many MAC standards including IEEE802.11 has been collision avoidance while optimizing the design of parameters based on a layered approach. Time Division Multiple Access (TDMA) and similar slotted access methods attempt at completely eliminating collisions by assigning orthogonal resources to different terminals. In heavy load conditions, slotted access methods use resources efficiently while in low load conditions, they waste unused slots. In contrast to slotted access methods, random access strategies such as Carrier Sense Multiple Access/Collision Avoidance (CSMA/CA) avoid collisions by sensing channel before transmission and randomizing the transmission time of the different nodes. In low load conditions, random access methods offer high throughput and spectral efficiency. However, collisions happen more often and spectral efficiency decreases for the latter methods as the load increases. There have been efforts to enhance spectral efficiency and improve the throughput of a network by offering hybrid design methods in the MAC layer. Hybrid MAC design methodology was first proposed in [2]. Later, Z-MAC [3] was proposed as a sensor network MAC protocol combining the strengths of TDMA and CSMA/CA. Recently, LA-MAC [4] was proposed as a MIMO-aware MANET MAC protocol capable of adaptively switching between TDMA and CSMA/CA modes of operation depending on real-time load conditions.

Recently, the introduction of Multiple-Input MultipleOutput (MIMO) systems along with cross-layer design methodologies have opened many different doors toward improving the efficiency and reliability of wireless communi-

This work was partially sponsored by a grant from the Boeing Company. cations. Generally speaking, layered approaches ignore the PHY layer parameters and their impact in the design of MAC algorithms. Traditional MAC design for WLANs usually avoids collisions and transmission of more than one packet at a time over the same medium. MIMO has transformed the traditional view.

MIMO assisted communication is able to resolve the problems associated with colliding transmissions. An $N$ antenna receiver can detect up to $N$ independent streams of data using V-BLAST [5] technique. There are array processing techniques which provide diversity in addition to simple multi-packet detection capability for MIMO systems [6], [7]. This motivates the design of MAC/PHY cross-layer algorithms leveraging a multiple-packet detection capability. In [8], the authors introduce a multi-packet reception MAC/PHY algorithm for ad-hoc networks which basically utilizes V-BLAST. For WLANs, the authors of [9] propose a joint multi-packet detection MAC and adaptive resource allocation algorithm.

In this paper, we introduce a novel cross-layer MAC/PHY multi-packet detection algorithm based on the interference cancellation technique introduced in [6]. Our proposal is based on the IEEE802.11 standard operating in DCF mode. It uses the same signaling sequence of RTS/CTS/DATA/ACK with minor modifications in the structure of CTS and ACK packets. We will analyze the throughput performance of the new MAC algorithm for a WLAN network in lossy channels and compare it to that of IEEE802.11 DCF mode calculated in [10]. Our analysis and matching simulation results illustrate the great potential of cross-layer MAC/PHY algorithms leveraging multi-packet detection to improve the rate of packet delivery in wireless networks.

The organization of this paper is as follows. In Section II, we introduce our new cross-layer algorithm. Section III presents the saturation throughput analysis of a WLAN leveraging our cross-layer MAC/PHY algorithm. Section IV presents the results of our theoretical analysis and compares those results with simulation results. Finally, Section V provides the conclusion of this paper.

\section{Multi-Packet Detector MAC}

In this section, we describe our multi-packet detector MAC algorithm. We note that our discussion revolves around a typical random topology of nodes utilizing an Access Point (AP) and forming a WLAN. As we are discussing MIMO systems, all nodes including the AP are assumed to be equipped with 
2 antennas and code their data using the Alamouti space-time code [11].

For the general case of $N$-antenna synchronized terminals employing Space-Time Block Codes (STBCs), a linear array processing algorithm is proposed in [6] to detect up to $N$ simultaneous transmissions in the PHY layer. This algorithm trades diversity for interference cancellation. An $N$-transmit $N$-receive MIMO system provides a diversity gain of $N \times N$, while using an array processing technique to detect $J \leq N$ signals will provide a diversity gain of $N(N-J+1)$. Briefly discussing, in the special case of a network of two-antenna terminals, when two synchronized terminals transmit simultaneously, the received signal at the $i$-th antenna of a receiving terminal can be represented as:

$$
\left(\begin{array}{c}
Y_{1 i} \\
Y_{2 i}^{*}
\end{array}\right)=\sum_{j=1}^{2}\left(\begin{array}{cc}
h_{1 i}^{j} & h_{2 i}^{j} \\
h_{1 i}^{j *} & -h_{1 i}^{j *}
\end{array}\right)\left(\begin{array}{c}
S_{1}^{j} \\
S_{2}^{j}
\end{array}\right)+\mathbf{N}
$$

where $S^{j}=\left(\begin{array}{ll}S_{1}^{j} & S_{2}^{j}\end{array}\right)^{T}$ is the transmitted signal of the $j$-th transmitting terminal and $\left\{h_{l i}^{j}\right\}_{i, l=1,2}^{j=1,2}$ are the corresponding Rayleigh channel coefficients, which are assumed to be i.i.d. Complex Gaussian variables. A simple array processing [6] on the received signals of the 2 receiver's antennas results in the following signal:

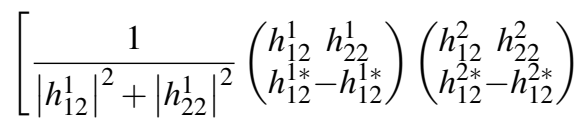

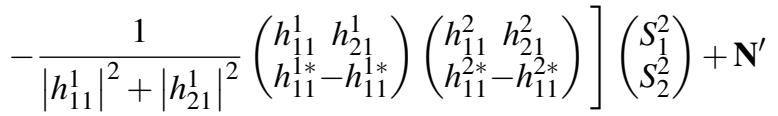

where the matrix at the left side of the second terminal's signal is a unitary matrix. Note that in Eq. (2), the signal of the first terminal has been canceled. Decoding the data of the second terminal from Eq. (2) is straightforward. A similar procedure can also be used to decode first terminal's data.

Now, suppose we have $n$ active terminals in the network. At a transmission instance, receiver decides how to decode the received signal based on the number of terminals transmitting. If one terminal transmits, the data of the transmitting terminal will be decoded as a 2-by-2 MIMO signal. This decoding process for a single user provides a diversity order of 4 . If two terminals transmit, the receiver uses the array processing technique discussed previously to eliminate interference and decode each terminals's data. We call this technique doublepacket detection. Double-packet detection provides diversity order of 2 for both terminals. In the case, more than two terminals transmit, the receiver cannot decode data from any of the transmitters and all transmissions will fail since all nodes are assumed to have only 2 antennas.

We will consider a WLAN that complies with the synchronization requirement of the double-packet detection method. Such a synchronization is achievable in a centralized topology. We assume that the WLAN has a single AP and that all nodes are in the hearing region of the AP. We also assume that all of the nodes are in the hearing range of each other, i.e., there is no hidden terminal problem, as in [10], [12]. Our multi-packet detector MAC algorithm improves the design of IEEE802.11 operating in DCF mode [1].

The IEEE802.11 standard operating in DCF mode relies on the sequence of RTS/CTS/DATA/ACK for signaling. For our double-packet detector MAC algorithm, the signaling sequence remains the same. In the normal IEEE802.11 standard in DCF mode, to which we refer as the single-packet detector MAC, the AP may be in three different states: (i) idle, (ii) receiving a successful single transmission, and (iii) hearing a collision. Since nodes may receive 2 simultaneous packets when utilizing double-packet detector MAC, the AP may be in one of the following four states: (i) idle, (ii) receiving a successful single transmission, (iii) receiving successful double transmissions, and (iv) hearing a collision. The first two states are the same as the states of the single-packet detector MAC, but the collision state is different. In the double-packet detector MAC, the PHY layer can resolve two simultaneous transmissions. Therefore, collisions happen when more than 2 terminals transmit their RTS packets simultaneously. Thus, simultaneous reception of 2 RTS packets represents a separate state. In the double reception state, the MAC layer will be specifically signaled by the PHY layer that 2 separate packets have been received correctly. Then, the PHY layer passes the two packets up in the layered stack for further processing. If the two detected packets are RTS packets, the addresses of the corresponding transmitters will be read from them. To construct the proper CTS packet in response, these addresses are needed. If the two detected packets are DATA packets, a proper ACK packet will be constructed in the MAC layer and DATA frames will be passed to the layers above the MAC layer.

Although the sequence of the RTS/CTS/DATA/ACK signals are the same for the double-packet detector MAC, the structure of the signals is slightly modified to account for doublepacket detection. In the case of double-packet detection, the AP needs to notify 2 terminals that either their RTS or DATA packets were received correctly. Hence, an extra address field with the size of 6 Bytes is added to CTS and ACK frames of IEEE802.11 standard. If the AP detects two correct simultaneous RTS (DATA) packets, it fills two address fields in a single CTS (ACK) packet with the addresses of the terminals transmitting RTS and sends it for transmission over the air. In the case of single packet detection, the second address field will be filled with a globally known AP address.

\section{Throughrut ANALYSIS}

In this section, we calculate the saturation throughput of a WLAN utilizing double-packet detector MAC in the absence of any hidden terminal problem. By saturation throughput, we mean that all terminals always have data ready to be transmitted. To calculate the saturation throughput of this network, we use an algorithm based on virtual slot definition proposed in [12], [10]. A virtual slot is the time interval between two consecutive decrements of a backoff counter 


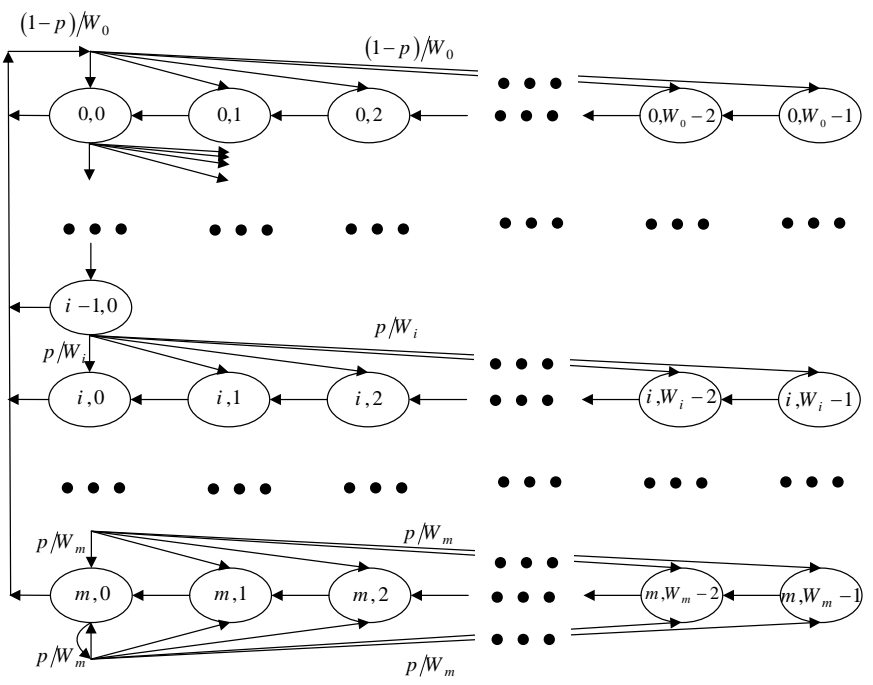

Fig. 1. The Markov Chain model used for the backoff counter.

for a terminal. Decrements of the backoff counter may be separated by an idle time slot when the channel is free or a complete transmission interval when the channel is busy. There are several types of virtual slots with different durations. In what follows, we go through two steps to calculate the saturation throughput of a WLAN with double-packet detector MAC. First, each terminal is modeled by a two-dimensional Markov Chain. Using the Markov model, we calculate the probability of transmission in each virtual slot. The next step is to categorize virtual slots and assign proper probabilities to each type of virtual slot.

\section{A. Transmission Probability}

A terminal's backoff counter variation can be represented by a two-dimensional Markov Chain [12] as illustrated by Fig. 1. Each state corresponds to the backoff counter value and its stage. In Stage $i$ with $i=0,1, \ldots, m$, the backoff counter value can be randomly chosen from $\left\{0, \cdots, W_{i}-1\right\}$ where $W_{i}=2^{i} W$ and $W$ is the minimum backoff counter size. In Fig. $1, p$ is the conditional probability of failure for a packet transmission. If we call $P(i, k \mid j, l)$ the probability of being in State $(i, k)$ given that in the last virtual slot we were in State $(j, l)$, we will have the following equations from the Markov Chain in Fig. 1:

$$
\begin{cases}P(i, k \mid i, k+1)=1 & k \in\left[0, W_{i}-2\right], i \in[0, m] \\ P(0, k \mid i, 0)=\frac{1-p}{W_{0}} & k \in\left[0, W_{i}-1\right], i \in[0, m] \\ P(i, k \mid i-1,0)=\frac{p}{W_{i}} & k \in\left[0, W_{i}-2\right], i \in[1, m] \\ P(m, k \mid m, 0)=\frac{p}{W_{m}} & k \in\left[0, W_{i}-2\right]\end{cases}
$$

While the backoff counter is non-zero, terminals are in the backoff state and do not transmit. Each terminal starts its transmission when its counter reaches any of the states $(i, 0)$. Therefore, $\tau$ the probability of transmission in a virtual slot can be written from Eq. (3) as:

$$
\tau=\frac{2(1-2 p)}{(1-2 p)(W+1)+p W\left(1-(2 p)^{m}\right)}
$$

To calculate the conditional failure probability, note that a packet transmission may fail due to collision or bit errors. Therefore, the conditional probability of failure, $p$, is given by:

$$
p=P_{C}+P_{e 1} P_{1}+P_{e 2} P_{2}
$$

where $P_{C}$ is the conditional collision probability, $P_{e 1}$ and $P_{e 2}$ are conditional error probabilities in the cases of single and double transmission, respectively. As mentioned in Section II, the array processing technique decreases the diversity order. Space-time coding over a 2-by-2 MIMO system provides a diversity order of 4 , while the double-packet detection technique presented in Section II has a diversity order of 2. Therefore, under fixed channel conditions, the double-packet detection has a higher bit error rate (BER) than that of the singlepacket detection. This means when two terminals transmit simultaneously, BER for CTS and ACK packets are different from the BER of RTS and DATA packets. We call the former BER $P_{b e r}^{1}$ and the latter BER $P_{b e r}^{2}$. We also define $P_{c 1}=1-P_{b e r}^{1}$ and $P_{c 2}=1-P_{b e r}^{2}$ as the probability of detecting a correct bit for CTS (ACK) packets and RTS (DATA) packets, respectively. We call $P_{1}$ the conditional probability that only the terminal under investigation transmits, and $P_{2}$ the conditional probability that exactly one terminal other than the one under investigation transmits. These probabilities are calculated in the following equations:

$$
\begin{aligned}
& P_{C}=1-(1-\tau)^{n-1}-(n-1) \tau(1-\tau)^{n-2} \\
& P_{1}=(1-\tau)^{n-1} \\
& P_{2}=(n-1) \tau(1-\tau)^{n-2} \\
& P_{e 1}=1-\left(1-P_{b e r}^{1}\right)^{R T S+C T S+H+L+A C K} \\
& P_{e 2}=1-\left(1-P_{b e r}^{2}\right)^{2 R T S+H+L}\left(1-P_{b e r}^{1}\right)^{2 C T S+A C K} \\
& -\left(1-P_{b e r}^{2}\right)^{R T S}\left(1-P_{b e r}^{1}\right)^{C T S+H+L+A C K} \\
& \times\left(1-\left(1-P_{b e r}^{2}\right)^{R T S}+\left(1-P_{b e r}^{2}\right)^{R T S}\left(1-\left(1-P_{b e r}^{1}\right)^{C T S}\right)\right)
\end{aligned}
$$

By solving the two equations relating $p$ and $\tau$, Eqs. (4) and (5), we will have the conditional probability of transmission in each virtual slot, $\tau$, for a given backoff window size and a given number of active terminals.

\section{B. Throughput Calculation}

The probability of being in each type of virtual slots depends on the conditional probability of transmission, $\tau$. In what follows, we categorize virtual slots and calculate their probabilities.

The double-packet detector MAC has eight different types of virtual slots: 1) Idle slot: When the channel is free, nodes decrement their backoff counter after each time slot. 2) Collision slot: The transmission of more than 2 RTS causes collision in which case nodes wait for a period of EIFS before decrementing their backoff counter after hearing a collision. Since IEEE802.11 standard has not determined the timeout interval, we use EIFS as the timeout interval for collided packets and packets received with error. 3) RTS error slot: The AP might receive RTS packets with bit errors in which case it drops them without sending CTS and terminates the 
transmission. 4) CTS error slot: If the CTS packet is received with bit errors, the receiving node drops it without sending DATA packets. After waiting for a period of EIFS, the node will decrement its backoff counter if the channel is still free. 5) DATA error slot: If AP receives a DATA packet with errors, it will drop it without sending ACK packet. 6) ACK error slot: If the ACK packet is received with bit errors, it will be dropped and transmission is assumed unsuccessful. 7) Successful slot (single): If RTS/CTS/DATA/ACK packets of a single terminal are received correctly, the packet transmission is successful. 8) Successful slot (double): If RTS/CTS/DATA/ACK packets of two terminals are received correctly, the packet transmission is successful. The last two virtual slots have the same duration, but have different contributions to the throughput of the system. The virtual slots durations are listed below:

1) $T_{1}=\delta$

2) $T_{2}=R T S+\sigma+E I F S+\delta$

3) $T_{3}=R T S+\sigma+E I F S+\delta$

4) $T_{4}=R T S+\sigma+S I F S+C T S+\sigma+E I F S+\delta$

5) $T_{5}=R T S+\sigma+S I F S+C T S+\sigma+S I F S+H+L+\sigma+$ $E I F S+\delta$

6) $T_{6}=R T S+\sigma+S I F S+C T S+\sigma+S I F S+H+L+\sigma+$ $S I F S+A C K+\sigma+E I F S+\delta$

7) $T_{7}=R T S+\sigma+S I F S+C T S+\sigma+S I F S+H+L+\sigma+$ $S I F S+A C K+\sigma+D I F S+\delta$

8) $T_{8}=R T S+\sigma+S I F S+C T S+\sigma+S I F S+H+L+\sigma+$ $S I F S+A C K+\sigma+D I F S+\delta$

In the equations above, $\delta$ is a slot time duration as specified in the standard [1], $\sigma$ is the one way propagation delay, $L$ is data frame length, and $H$ is the header overhead. RTS, CTS, and $A C K$ are the duration of RTS, CTS, and ACK packets, respectively. Table I lists the parameters used in this paper to obtain numerical and simulation results including those of the Direct Sequence Spread Spectrum (DSSS) PHY specified by the IEEE802.11 standard.

Next, we calculate the probability of each virtual slot type. Denote $P_{\text {idle }}$ the probability of having an empty virtual slot, and $P_{t r}$ the probability of having transmission. These probabilities are given by:

$$
\begin{aligned}
P_{\text {idle }} & =(1-\tau)^{n} \\
P_{t r} & =1-(1-\tau)^{n}
\end{aligned}
$$

Other needed probabilities are $P_{s 1}$ the probability of single transmission without collision and $P_{s 2}$ the probability of double transmission without collision both conditioned on having at least one transmission. These two probabilities are specified below:

$$
\begin{aligned}
& P_{s 1}=\frac{n \tau(1-\tau)^{n-1}}{P_{t r}}=\frac{n \tau(1-\tau)^{n-1}}{1-(1-\tau)^{n}} \\
& P_{s 2}=\frac{\frac{n(n-1)}{2} \tau^{2}(1-\tau)^{n-2}}{P_{t r}}=\frac{\frac{n(n-1)}{2} \tau^{2}(1-\tau)^{n-2}}{1-(1-\tau)^{n}}
\end{aligned}
$$

Since all nodes hear each other, the variation of their virtual slots will be the same and the same as that of the AP. We define throughput as the number of packets delivered to the
TABLE I

SYSTEM PARAMETERS

\begin{tabular}{|l|c|}
\hline Propagation Delay & $1 \mu \mathrm{s}$ \\
\hline SIFS & $10 \mu \mathrm{s}$ \\
\hline SlotTime & $20 \mu \mathrm{s}$ \\
\hline DIFS & SIFS+2SlotTime \\
\hline MAC header & 272 bits \\
\hline PHY header & 192 bits \\
\hline H & MAC header+PHY header \\
\hline RTS & $160+$ MAC header bits \\
\hline CTS & $122+(48)+$ MAC header bits \\
\hline ACK & $122+(48)+$ MAC header bits \\
\hline EIFS & SIFS+CTS(ACK)+DIFS \\
\hline Data(L) & $\{256,512,1024\}$ \\
\hline Data Rate & 1 Mbps \\
\hline
\end{tabular}

AP per second. Therefore, we study the probability of virtual slots from the prospective of the AP. It is important to note that when the AP receives two simultaneous RTS packets, it sends a CTS packet if it receives at least one correct RTS packet. If one of the RTS packets is received correctly while the other one has bit errors, the virtual slot is not of Type 3. Rather, its type is defined by the correctly received RTS packet. For example, if the correctly received RTS is followed by successful transmission of CTS/DATA/ACK packets, the virtual slot will be of Type 7 . While the probability of each type of virtual slots is calculated below, the rest of equations are shown at the top of the next page.

$$
\begin{aligned}
& P_{1}=P_{\text {idle }} \\
& P_{2}=P_{t r}\left(1-P_{s 1}-P_{s 2}\right) \\
& P_{3}^{\text {single }}=P_{t r} P_{s 1}\left(1-P_{c 1}^{R T S}\right) \\
& P_{3}^{\text {double }}=P_{t r} P_{s 2}\left(1-P_{c 2}^{R T S}\right)^{2} \\
& P_{4}^{\text {single }}=P_{t r} P_{s 1} P_{c 1}^{R T S}\left(1-P_{c 1}^{C T S}\right) \\
& P_{5}^{\text {single }}=P_{t r} P_{s 1} P_{c 1}^{R T S+C T S}\left(1-P_{c 1}^{H+L}\right) \\
& P_{6}^{\text {single }}=P_{t r} P_{s 1} P_{c 1}^{R T S+C T S+H+L}\left(1-P_{c 1}^{A C K}\right) \\
& P_{7}^{\text {single }}=P_{t r} P_{s 1} P_{c 1}^{R T S+C T S+H+L+A C K} \\
& P_{8}=P_{t r} P_{s 2} P_{c 2}^{2 R T S+2(H+L)} P_{c 1}^{2 C T S+2 A C K}
\end{aligned}
$$

In Eq. (8), $P_{i}=P_{i}^{\text {single }}+P_{i}^{\text {double }}$ where $P_{i}^{\text {single }}$ and $P_{i}^{\text {double }}$ are the probabilities of being in the Type $i$ virtual slot while the RTS packet is being decoded by single-packet or doublepacket detection technique, respectively. As mentioned before, the virtual slot type depends on the longer lasting transmission when 2 terminals transmit their RTS packets together. Therefore, we need to consider different scenarios to calculate the probability of each virtual slot when double-packet detection is used to detect RTS packets.

The definition of throughput suggests that the throughput of 


$$
\begin{aligned}
P_{4}^{\text {double }}= & P_{t r} P_{s 2}\left(P_{c 2}^{2 R T S}\left(1-P_{c 1}^{2 C T S}\right)^{2}+2 P_{c 2}^{R T S}\left(1-P_{c 1}^{C T S}\right)\left(1-P_{c 2}^{R T S}\right)\right) \\
P_{5}^{\text {double }}= & P_{t r} P_{s 2}\left(P_{c 2}^{2 R T S} P_{c 1}^{2 C T S}\left(1-P_{c 2}^{(H+L)}\right)^{2}+2 P_{c 2}^{2 R T S} P_{c 1}^{C T S}\left(1-P_{c 1}^{C T S}\right)\left(1-P_{c 1}^{H+L}\right)+2 P_{c 2}^{R T S} P_{c 1}^{C T S}\left(1-P_{c 2}^{R T S}\right)\left(1-P_{c 1}^{H+L}\right)\right) \\
P_{6}^{\text {double }}= & P_{t r} P_{s 2} P_{c 2}^{R T S} P_{c 1}^{C T S}\left(1-P_{c 1}^{A C K}\right)\left(P_{c 2}^{R T S+2 H+2 L} P_{c 1}^{C T S}\left(1-P_{c 1}^{A C K}\right)+2 P_{c 2}^{R T S} P_{c 1}^{C T S}\left(1-P_{c 2}^{H+L}\right) P_{c 2}^{H+L}+2 P_{c 2}^{R T S}\left(1-P_{c 1}^{C T S}\right) P_{c 1}^{H+L}\right. \\
& \left.+2\left(1-P_{c 2}^{R T S}\right) P_{c 1}^{H+L}\right) \\
& P_{7}^{\text {double }}= \\
& 2 P_{t r} P_{s 2} P_{c 2}^{R T S} P_{c 1}^{C T S+A C K}\left(P_{c 2}^{R T S+H+L} P_{c 1}^{C T S}\left\{P_{c 2}^{H+L}\left(1-P_{c 1}^{A C K}\right)+\left(1-P_{c 2}^{H+L}\right)\right\}+P_{c 1}^{H+L}\left\{P_{c 2}^{R T S}\left(1-P_{c 1}^{C T S}\right)+\left(1-P_{c 2}^{R T S}\right)\right\}\right)
\end{aligned}
$$

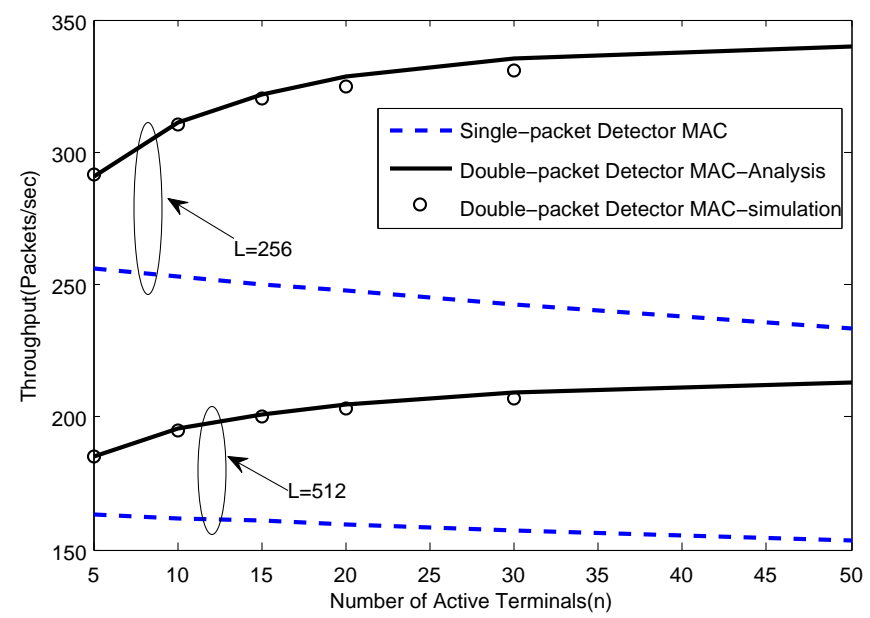

Fig. 2. The throughput of double-packet detector MAC algorithm versus the throughput of IEEE802.11 DCF for different number of active nodes with $W=32$ and $m=3$.

a double-packet detector network is expressed as:

$$
S=\frac{\left(P_{7}+2 P_{8}\right)}{\sum_{i=1}^{8} P_{i} T_{i}}
$$

The throughput of the single-packet detector MAC can be easily calculated from the same set of equations. The only modifications required are to set $P_{C}=1-(1-\tau)^{n-1}$ and $P_{e 2}=P_{s 2}=0$.

\section{Numerical Results}

To investigate the performance of the double-packet detector MAC, we set up a network of one two-antenna AP and $n$ active two-antenna terminals which use the double-packet detector MAC algorithm as described in Section II. The throughput performance is compared to the throughput performance of IEEE802.11 standard in DCF mode in a network of one twoantenna AP and $n$ active single-antenna terminals. Terminals consume the same amount of power for transmission in both networks and we ignore the synchronization overhead for the double-packet detector MAC and compare MAC throughput. Other parameters used for the simulation and numerical results are listed in Table I.

The curves in Fig. 2 represent the throughput of the singlepacket detector MAC and the double-packet detector MAC for data packet lengths of $256 B$ and $512 B$. Eqs. (5) and (4) provide

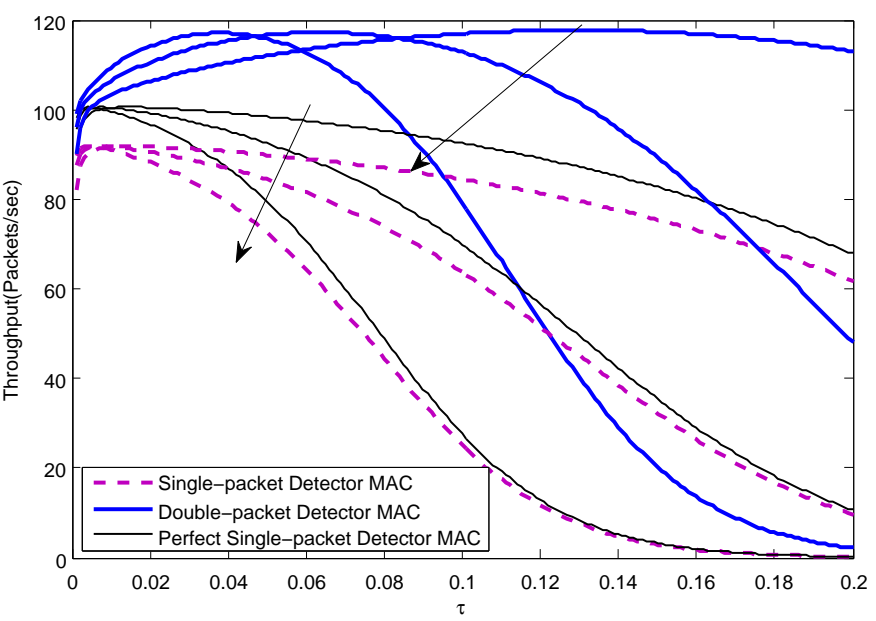

Fig. 3. Comparison of throughput for new MAC algorithm and IEEE802.11 DCF for different transmission probabilities, $W=32, m=3, L=1024 B$, and $\mathrm{n} \in\{15,30,50\}$. The direction of the arrows shows the increase in the value of $n$.

the corresponding probability of transmission. From Eq. (9), the saturation throughput of the network is calculated. In order to validate the analytical results, NS2 is used to simulate a WLAN of one AP and $n$ nodes all using our proposed doublepacket detector MAC. The IEEE802.11 module of NS2 is modified to support the double-packet detector MAC. Since NS2 simulator does not directly implement the PHY layer, and our MAC module is aware of multi-packet reception, we implement the packet drop event for a fixed BER in the MAC module. We use a Constant Bit Rate (CBR) traffic pattern in our NS2 experiments. Each simulation point in the graph represents 5 independent runs of the traffic scenario for 300 seconds of simulation time. Other simulation parameters are listed in Table I. As illustrated by the figure, the results of simulation match the analytical results. From Fig. 2, it is clear that double-packet detector MAC enhances the throughput performance of the network. Single- and double-packet detector MACs have different behaviors when the number of competing terminals increases. The former's throughput decreases because of the higher collision probability and the latter's increases because of the higher probability of doublepacket detection.

In Fig. 3, we use Eq. (9) to plot the throughput of the network versus $\tau$ the transmission probability for both 


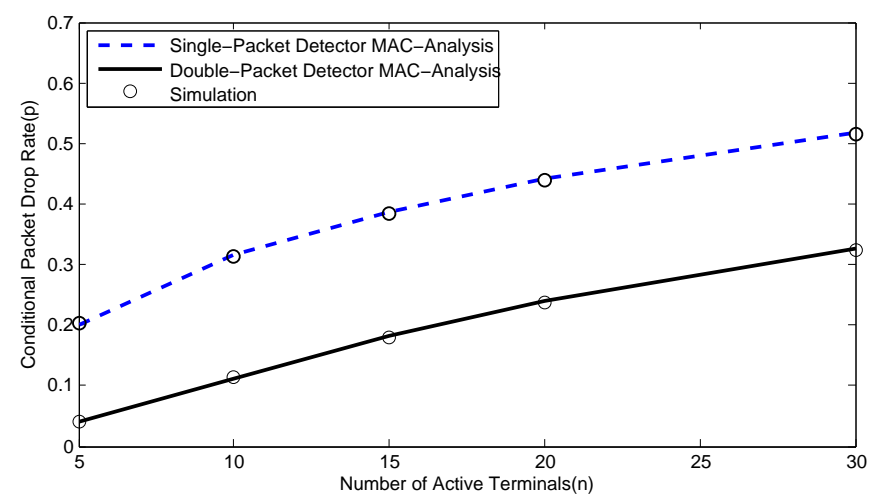

Fig. 4. A comparison of conditional packet loss rate in single- and doublepacket detector MAC, with $W=32, m=3, L=256 B$

IEEE802.11 DCF and double-packet detector MACs. The performance of perfect (error free) single-packet detector MAC is also presented to illustrate the limiting effects of collision on the throughput in single-packet detector networks. Multipleantenna nodes consuming the same amount of power as singleantenna nodes, provide lower BER. The curves of perfect single-packet detector MAC show that the throughput gain of double-packet detector MAC can not be achieved only by using multiple-antenna nodes. As Fig. 3 suggests, the doublepacket detector MAC outperforms the single-packet detector MAC specially when the probability of transmission increases. In single-packet detector MAC algorithms such as IEEE802.11 $\mathrm{DCF}$, increasing the probability of transmission beyond a certain point translates to a higher number of collisions. However in the double-packet detector MAC, collisions of two packets have the potential of becoming double successful transmissions. As expected, the double-packet detector MAC always performs better than IEEE802.11 DCF for different number of nodes and transmission probabilities as illustrated in Fig. 3.

In Fig. 4, conditional packet loss probability $p$ is plotted for both single- and double-packet detector MACs. Eq. (5) is used to calculate the conditional packet loss probability in the double-packet detector MAC. As shown by the figure, the results of simulation match those of analytical results. Number of active terminals is a representation of the load in the network. As the load increases, the possibility of collision between simultaneously transmitted packets in the single-packet detector MAC goes up. On the other hand, in the doublepacket detector MAC, double simultaneous transmissions are detected. This keeps the packet loss probability lower than that of the single-packet detector MAC. For example, when there are 30 active terminals in the network about $50 \%$ of transmissions fail using the single-packet detector MAC, while the failur rate in the double-packet detector MAC is about $30 \%$.

\section{CONCLUSION}

In this paper, we proposed a new cross-layer MAC/PHY algorithm which resolves the collision between two packets leveraging a novel interference cancellation method in the PHY layer. Then, we developed analytical models to characterize the saturation throughput of a WLAN using our proposed multi-packet detector MAC algorithm. The proposed analytical method took into account the bit error probability of packet transmission in both single- and double-packet detection modes. The results illustrated significant enhancements in the throughput of a WLAN. Extension of our MAC algorithm to users with more than 2 antennas using the interference cancellation methods in [6] is straightforward. We are currently working on the extension of our proposed multi-packet detector MAC algorithm to ad-hoc and multi-hop networks. As a part of our future work, we will be looking at addressing the details of synchronization issues as well as cross-layer routing schemes utilizing our proposed MAC algorithm.

\section{REFERENCES}

[1] IEEE 802.11 Standard - Part 11: Wireless LAN Medium Access Control (MAC) and Physical Layer (PHY) Specifications, Std., Jan. 2007.

[2] B. Sharp, E. Grindrod, and D. Camm, "Hybrid TDMA/CSMA protocol for self managing packet radio networks," 4th IEEE ICUPC '95, pp. 929-933, Nov. 1995.

[3] I. Rhee, A. Warrier, M. Aia, J. Min, and M. Sichitiu, "Z-MAC: A hybrid MAC for wireless sensor networks," IEEE/ACM Trans. on Networking, vol. 16, pp. 511-524, June 2008.

[4] W. Hu, X. Li, and H. Yousefi'zadeh., "LA-MAC: A load adaptive MAC protocol for MANETs," Proc. IEEE GLOBECOM (To Appear), Dec. 2009.

[5] P. Wolniansky, G. Foschini, G. Golden, and R. Valenzuela, "V-BLAST: An architecture for realizing very high data rates over the rich-scattering wireless channel," Proc. ISSSE-98, pp. 295-300, Sep. 1998.

[6] J. Kazemitabar and H. Jafarkhani, "Multiuser interference cancellation and detection for users with more than two transmit antennas," IEEE Trans. on Commun., vol. 56, pp. 574-583, Apr. 2008.

[7] A. Naguib, N. Seshadri, and A. Calderbank, "Applications of space-time block codes and interference suppression for high capacity and high data rate wireless systems," Proc. 32nd Asilomar Conf. Signals, Systems and Computers, vol. 2, pp. 1803-1810, Nov. 1998.

[8] P. Casari, M. Levorato, and M. Zorzi, "MAC/PHY crosslayer design of MIMO ad hoc networks with layered multiuser detection," IEEE Trans. on Wireless Commun., vol. 7, pp. 4596-4607, Nov. 2008.

[9] W. L. Huang, K. Letaief, and Y. J. Zhang, "Cross-layer multi-packet reception based medium access control and resource allocation for space-time coded MIMO/OFDM," IEEE Trans. on Wireless Commun., vol. 7, pp. 3372-3384, Sept. 2008.

[10] X. Dong and P. Varaiya, "Saturation throughput analysis of IEEE 802.11 wireless LANs for a lossy channel," IEEE Commun. Letters, vol. 9, pp. 100-102, Feb. 2005.

[11] S. Alamouti, "A simple transmit diversity technique for wireless communications," IEEE J. on Select. Areas in Commun., vol. 16, pp. 1451-1458, Oct. 1998.

[12] G. Bianchi, "Performance analysis of the IEEE 802.11 distributed coordination function," IEEE J. on Select. Areas in Commun., vol. 18, pp. 535-547, Mar. 2000. 